\title{
Performance estimation of a remote field eddy current method for the inspection of water distribution pipes
}

Sophie Duchesne - INRS Centre Eau Terre Environnement

Nabila Bouzida

Jean-Pierre Villeneuve

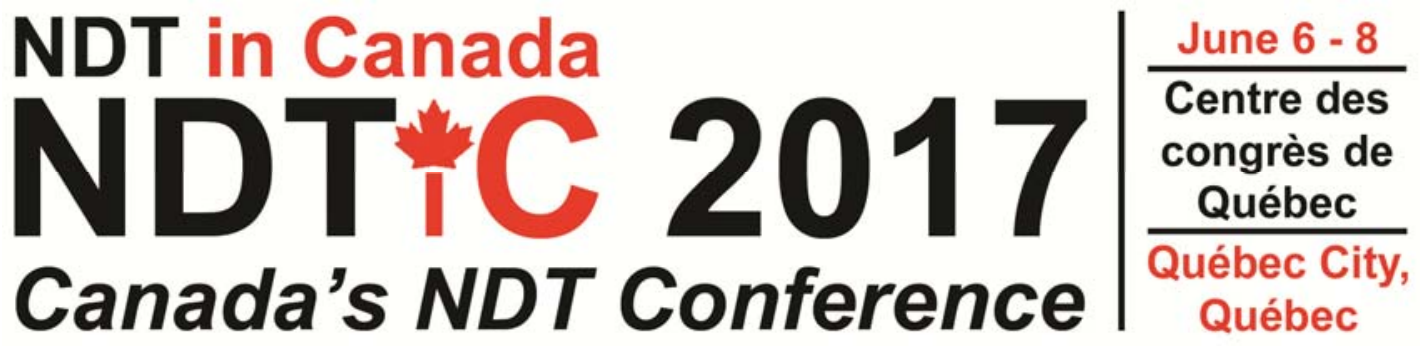




\section{Degradation and renewal of water distribution pipes}

- Replacement of water pipes: important expenditures

- e.g. Burn et al. (2007): annual worldwide expenditure for water distribution pipes > US $\$ 33,000$ million/year

- should rise significantly in the future as existing assets increasingly come to the end of their useful lives

- Most of small diameter pipes installed $<1990=$ metallic (ductile or gray cast iron)

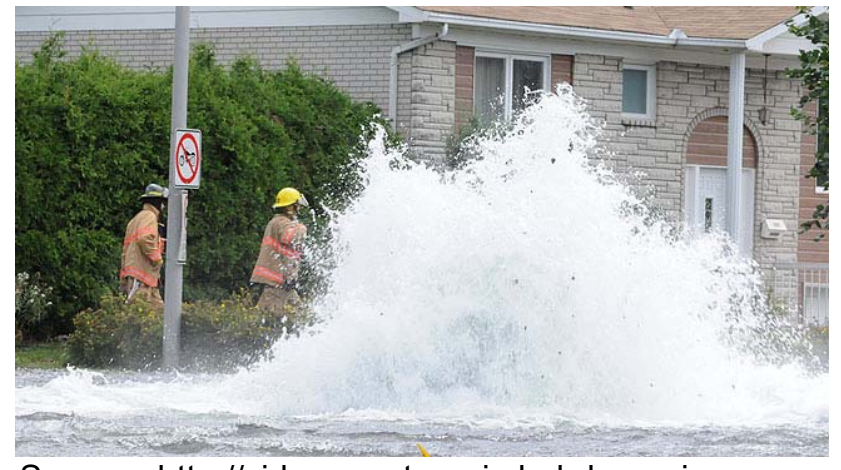

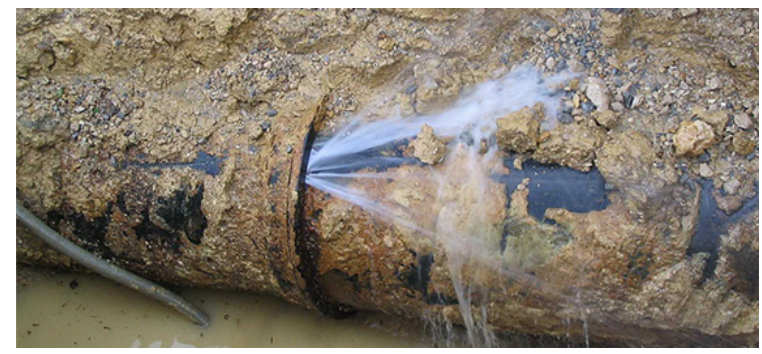

Source : http://www.cfgservices.fr 


\section{Corrosion of water distribution pipes}

Cast iron pipes (installation)
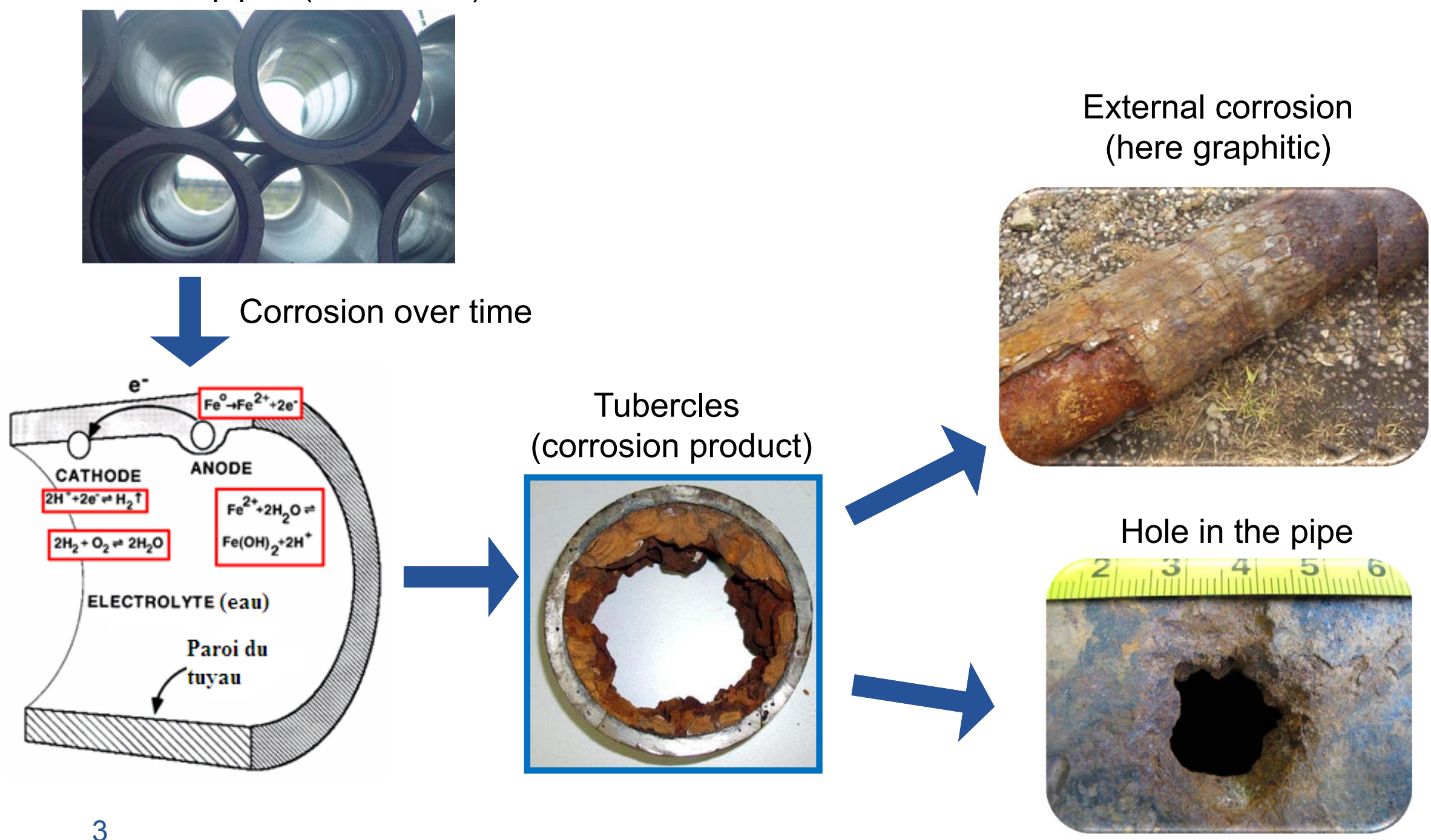


\section{Consequences of corrosion}

- Increased frequency / probability of pipe breaks and leaks

- Increased costs + interruptions in water supply

- Solutions:

- replace?

- repair?

- which pipes?

- when?
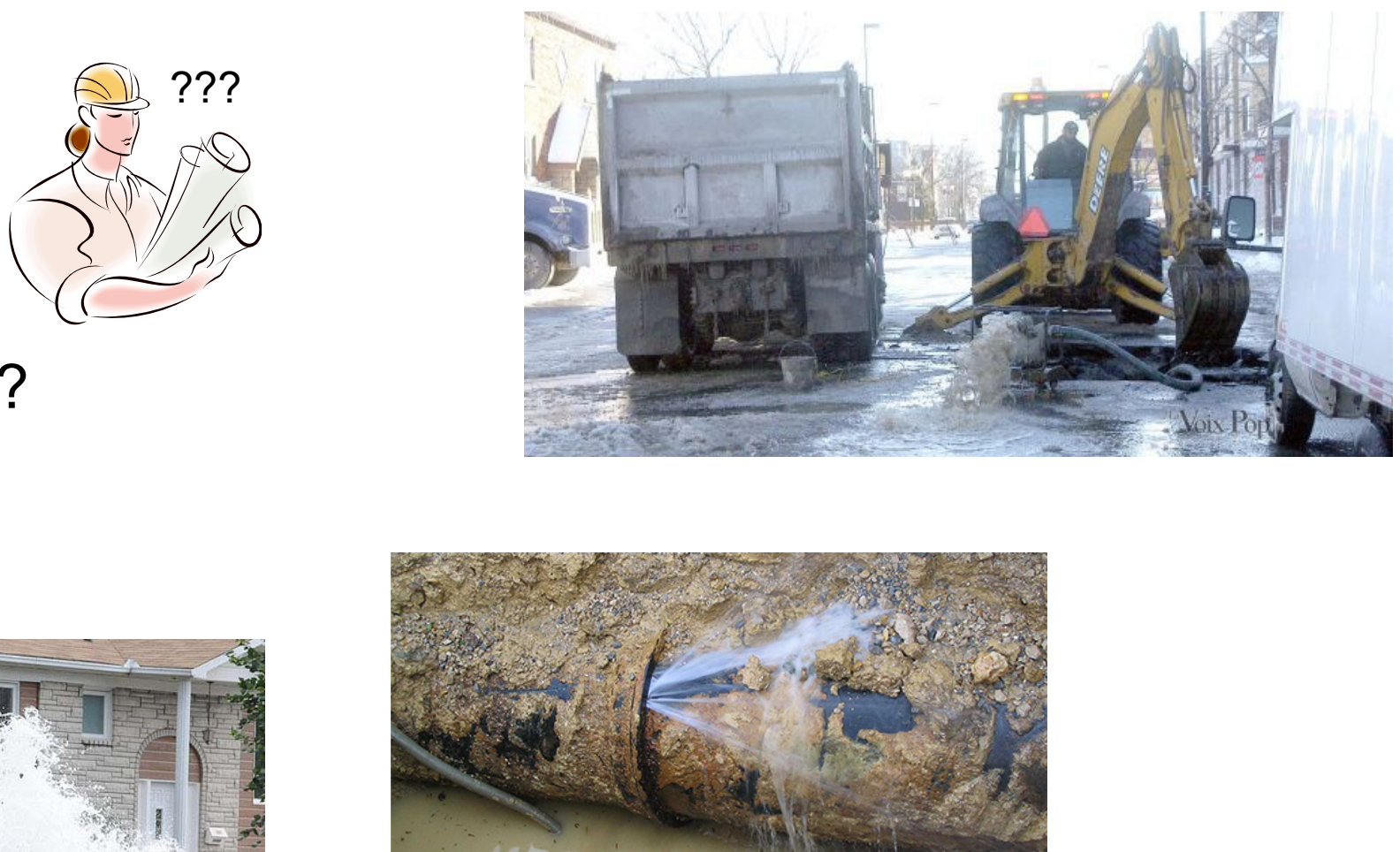

Source : http://www.cfgservices.fr 


\section{Existing tools to plan the renewal}

- Prediction models and planning tools:

- assess the required financial resources

- prioritizing pipes that should be replaced and/or rehabilitated

- Decision to replace / repair a specific pipe:

- requires assessment of its conditions

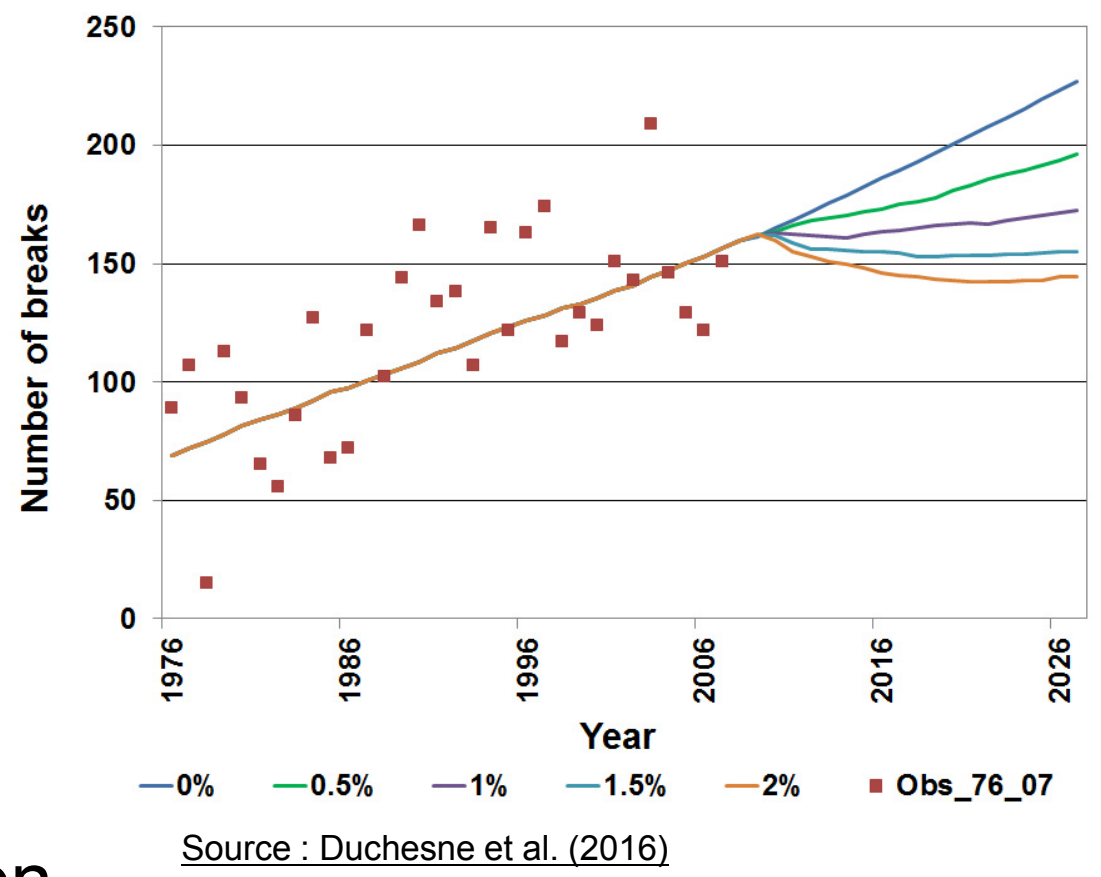

$>$ observations from inspection

$>$ observed breaks and leaks (indicators)

Source : Duchesne et al. (2016) 


\section{RFEC technique for the inspection of water distribution pipes}

- Remote Field Eddy Current:

- application well known for the identification and sizing of defects in metallic gas distribution pipes

- can be applied to water distribution pipes

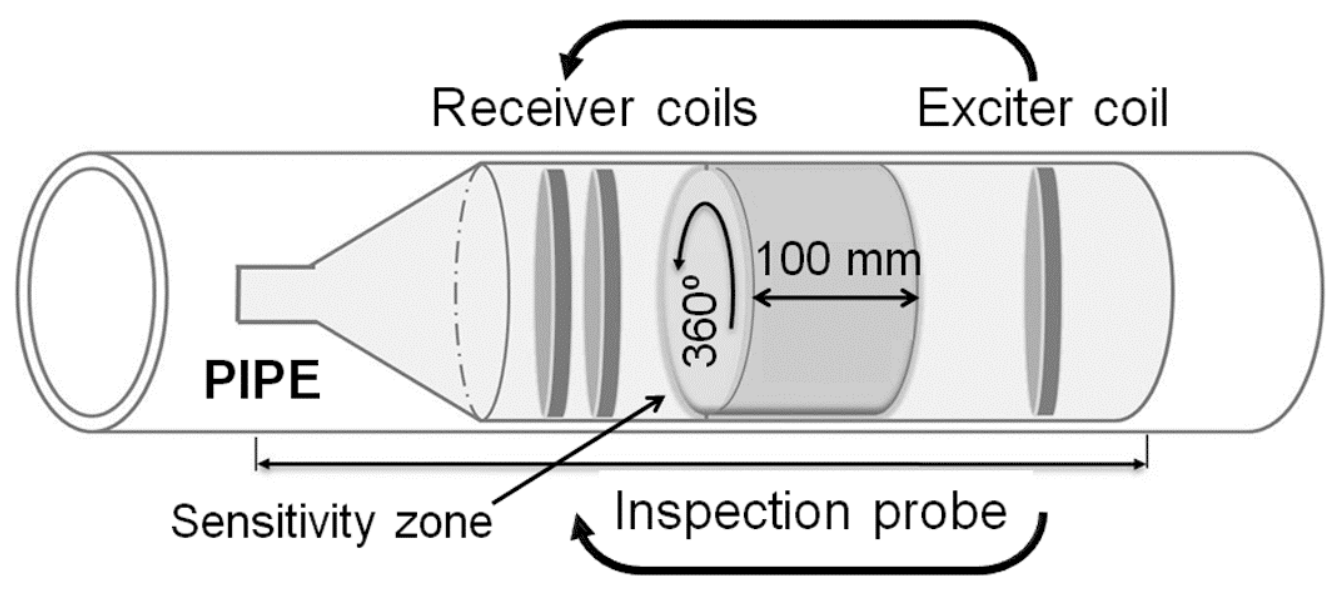

6

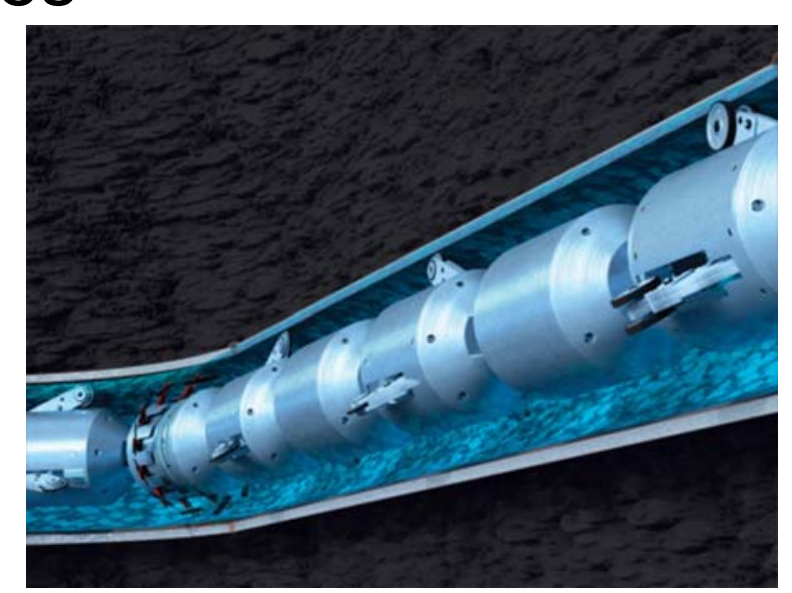

Source : http://www.popsci.com/technology/article/2011-01/pigrobots-keep-gas-lines-blowing 


\section{RFEC technique for the inspection of water distribution pipes}

- Exciter transmits a low frequency magnetic field that can reach receivers by two paths:

1. inside the pipe through the water (direct path)

2. through the outside of the pipe (indirect path)

- Strength of magnetic field attenuated rapidly in direct path

$>$ at $\approx$ two pipe diameters from exciter, indirect field dominates the direct field: the remote field zone begins

7

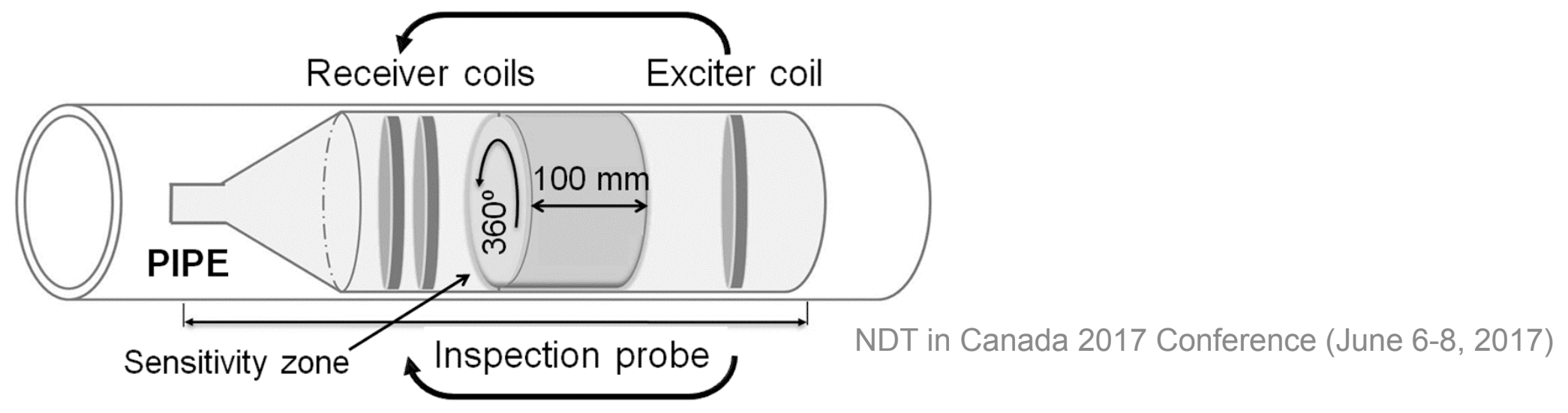




\section{RFEC technique for the inspection of water distribution pipes}

- Variations of wall thickness at the locations where the magnetic field goes through the pipe modify phase and/or amplitude of the signal

$>$ can be translated into wall thickness reduction and spatial extent of the detected flaw

- Does not measure the actual pipe-wall thickness: evaluation of the material loss percentage

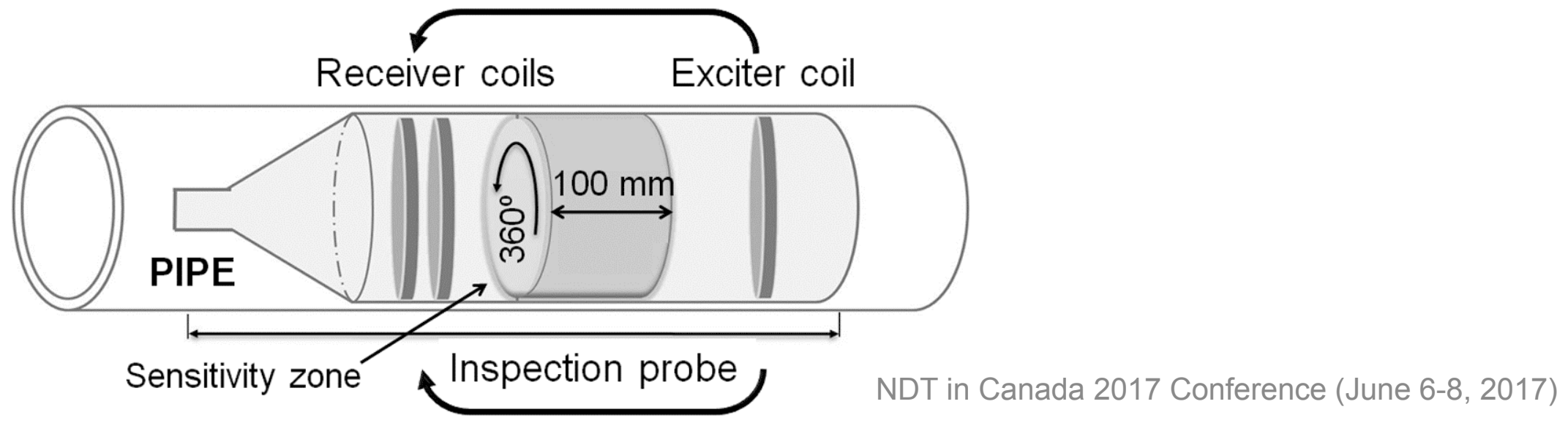




\section{Objective and general methodology}

Objective : Assess the performance of an existing RFEC probe for the inspection of cast iron water pipes

1. Inspect 6 pipes with the probe

2. Compare size and location of corrosion defects estimations with values resulting from the processing of computed tomography (CT) images of the same pipes

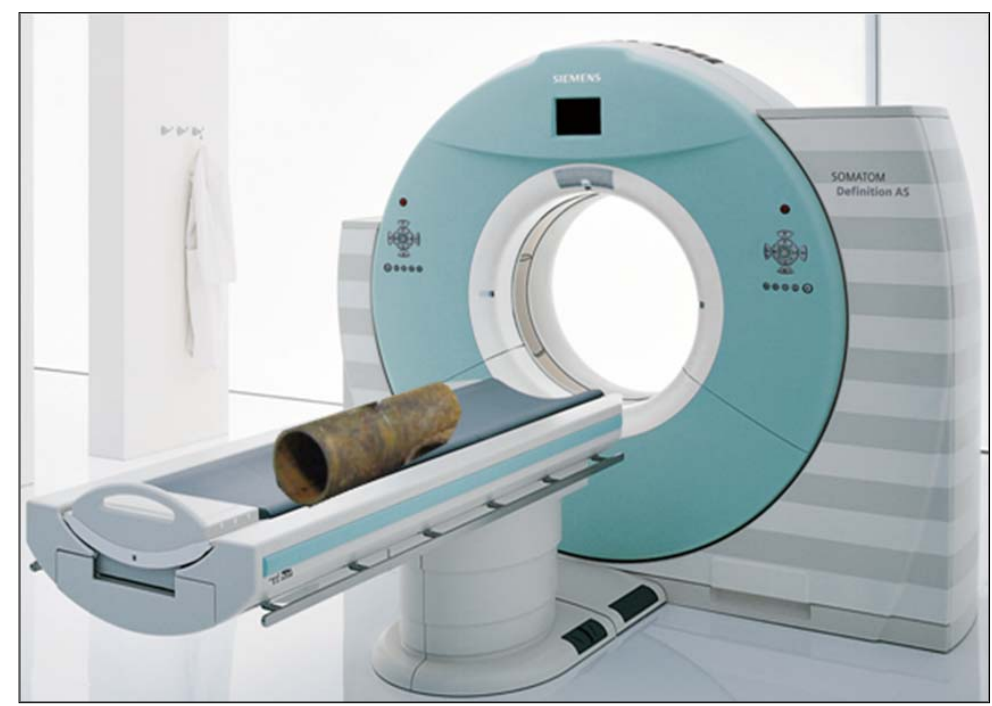




\section{Analyzed pipes (excavated)}

\begin{tabular}{l|c|c|c|c|c} 
& \multirow{2}{*}{$\begin{array}{c}\text { Estimated date } \\
\text { of installation }\end{array}$} & $\begin{array}{c}\text { Estimated age } \\
\text { at inspection }\end{array}$ & Diameter & Length & $\begin{array}{c}\text { Average wall } \\
\text { thickness }\end{array}$ \\
\cline { 3 - 6 } & - & $($ years $)$ & $(\mathrm{mm})$ & $(\mathrm{m})$ & $(\mathrm{mm})$ \\
\hline NEW-PIPE & 1948 & 61 & 150 & 1.1 & 7 \\
\hline SILL-MAG-1A & 1909 & 100 & 150 & 1.6 & 14 \\
\hline B-MAN-1A & 1909 & 100 & 150 & 2.5 & 10 \\
\hline B-MAN-1B & 1909 & 100 & 150 & 1.7 & 12 \\
\hline B-MAN-2A & 1909 & 100 & 150 & 1.7 & 9 \\
\hline B-MAN-2B & 1945 & 64 & 150 & 1.3 & 7 \\
\hline LHSTCH-MC & 1957 & 52 & 150 & 3.2 & 8 \\
\hline LHSTCH-HOP & & - & & 12 \\
\hline
\end{tabular}

- RFEC probe passed once in each pipe (laboratory = air)

- Comparison with in situ inspection for one pipe 


\section{Inspection results}

\begin{tabular}{l|c|c|c|c} 
& \multirow{2}{*}{ Defect } & Location & Thickness loss & $\begin{array}{c}\text { Sensitivity zone } \\
\text { coverage }\end{array}$ \\
\cline { 3 - 5 } & & $(\mathrm{m})$ & $(\%)$ & $(\%)$ \\
\hline B-MAN-1A & $\# 1$ & 1.2 & 22 & 22 \\
\hline B-MAN-1B & $\# 1$ & 0.8 & 17 & 13 \\
\hline B-MAN-2A & $\# 2$ & 1.6 & 28 & 17 \\
\hline B-MAN-2B & $\# 1$ & 1.8 & 15 & 63 \\
\hline LHSTCH-MC & $\# 1$ & 0.4 & 23 & 13 \\
\hline LHSTCH-HOP & $\# 1$ & 1.3 & 26 & 24 \\
\hline
\end{tabular}

11

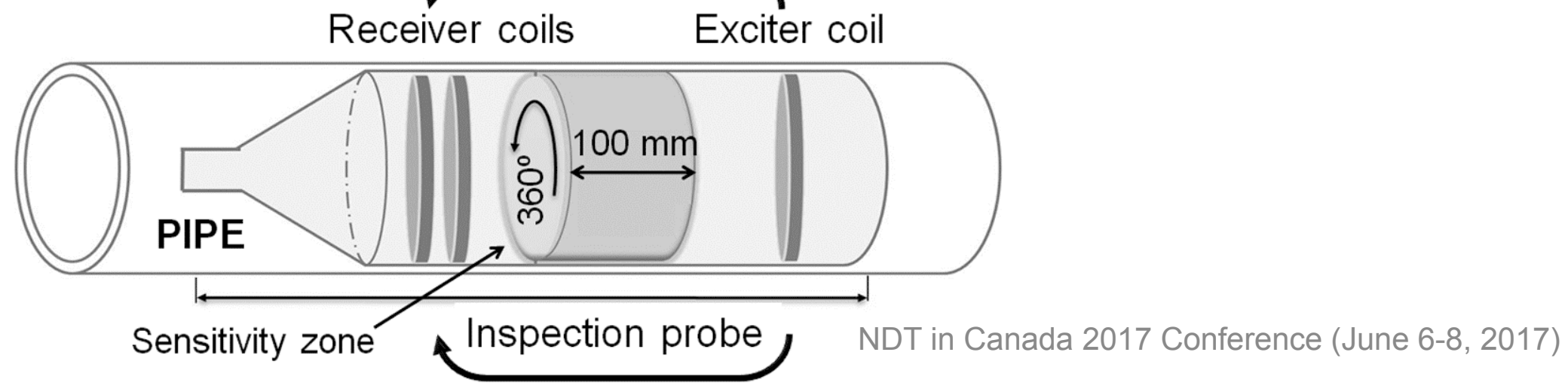




\section{Condition evaluation with the CT scan}

- Based on Lambert-Beer law:

$$
N=N_{0} e^{-\mu x}
$$

$N=$ measured intensity after layer of thickness $x$;

$N_{0}=$ incident radiation intensity (usually in $\mathrm{keV}$ );

$\mu=$ linear attenuation coefficient $\rightarrow$ depends linearly on the density of the material

- Output from CT scan computer: $H U=\frac{\mu-\mu_{\text {water }}}{\mu_{\text {water }}} \times 1000$

- When viewed in Matlab:

$$
\text { pixel_value }=\frac{H U+10240}{10}
$$




\section{Condition evaluation with the CT scan}
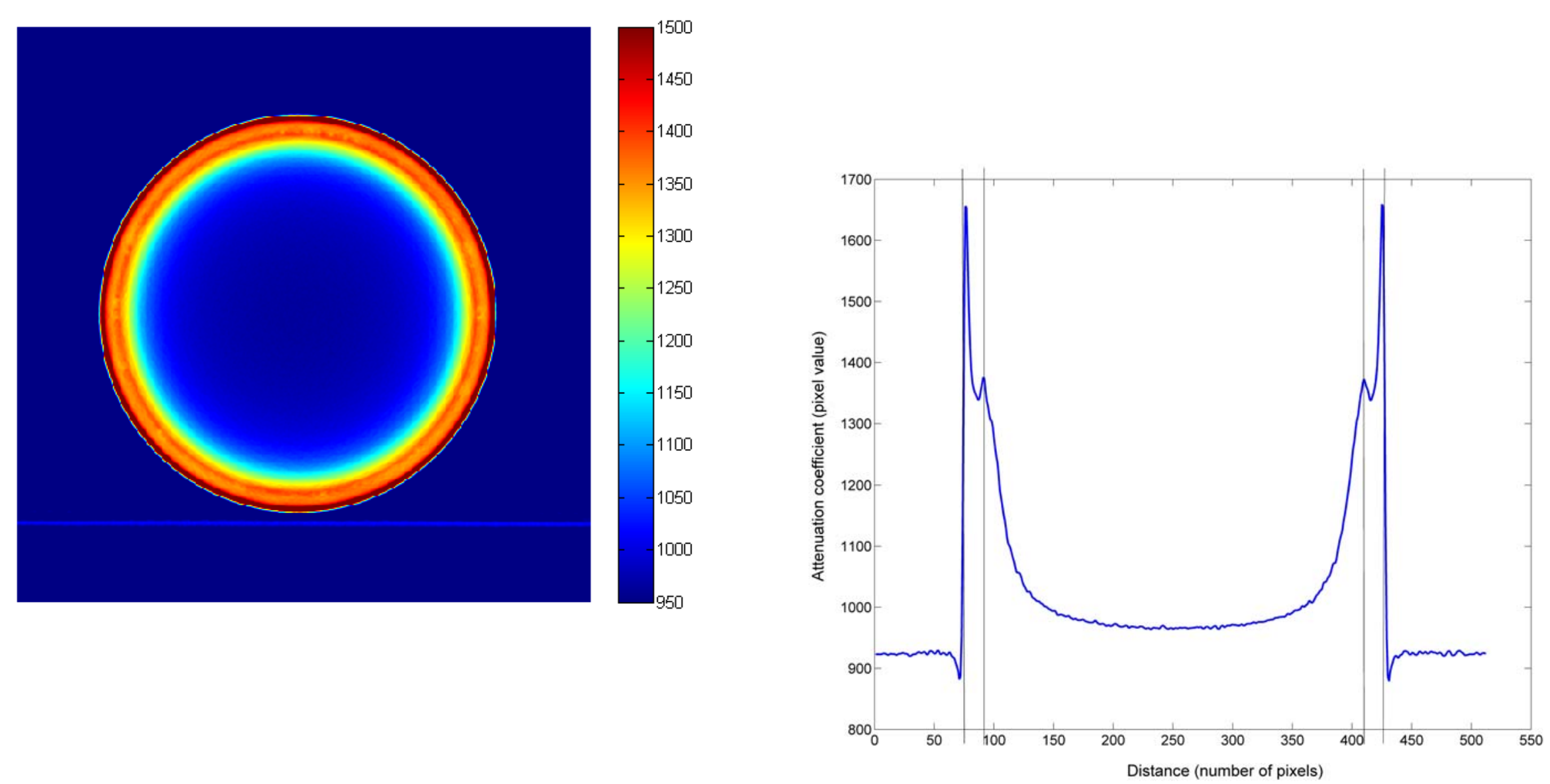

- When viewed in Matlab:

$$
\text { pixel_value }=\frac{H U+10240}{10}
$$




\section{Condition evaluation with the CT scan}

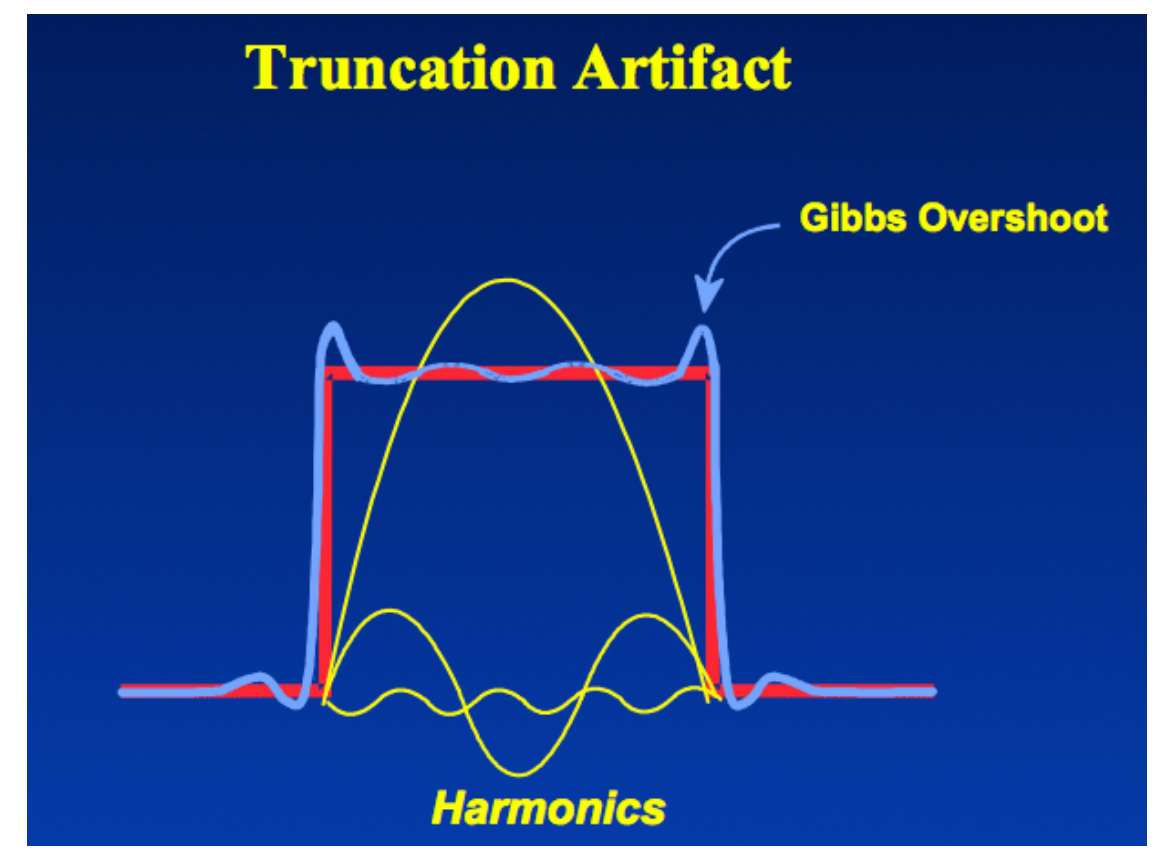

Source: http://mriquestions.com/gibbs-artifact.html

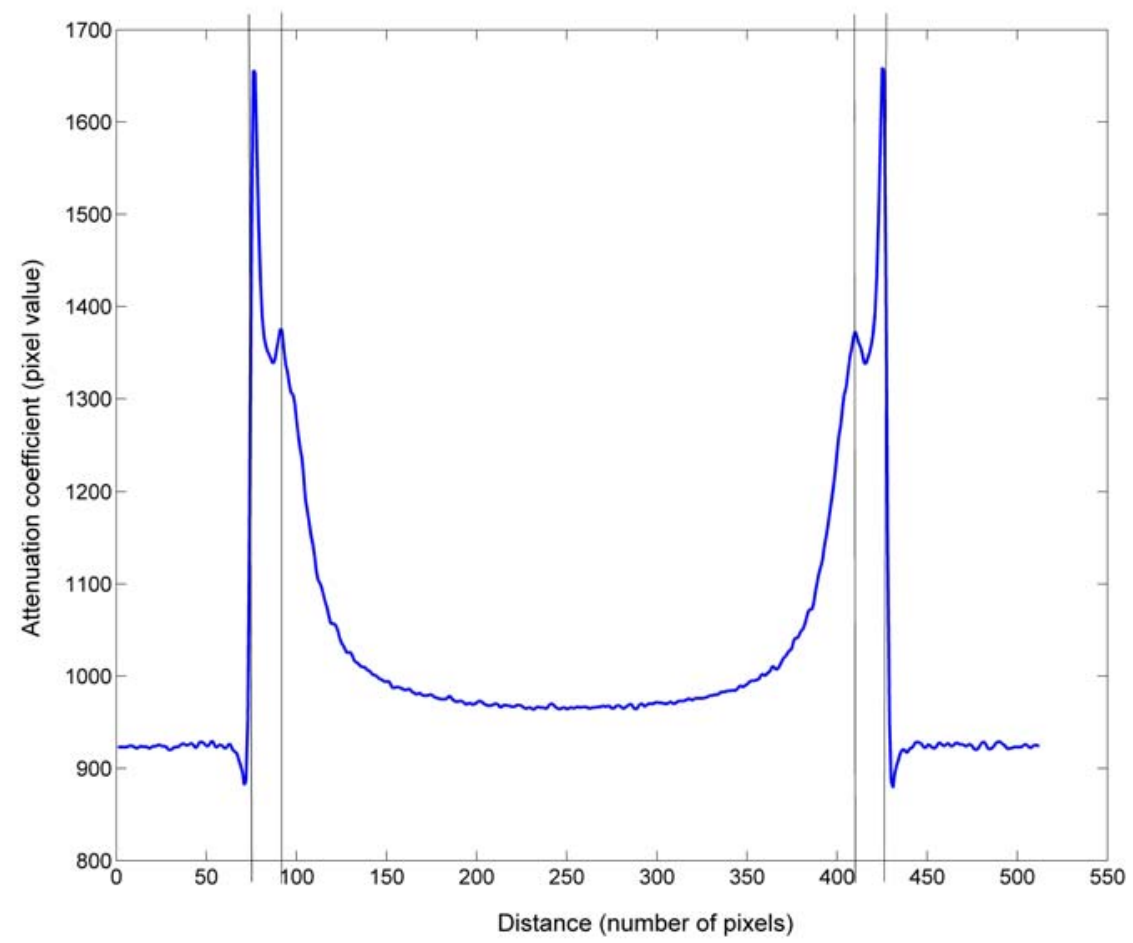

- When viewed in Matlab :

$$
\text { pixel_value }=\frac{H U+10240}{10}
$$




\section{Condition evaluation with the CT scan}

- Objective, to compare with the RFEC tool:

1. pipe thickness loss $=$ percentage of lost material on $100 \mathrm{~mm}$ sensitivity zones, all along the pipes

2. spatial extent of this loss

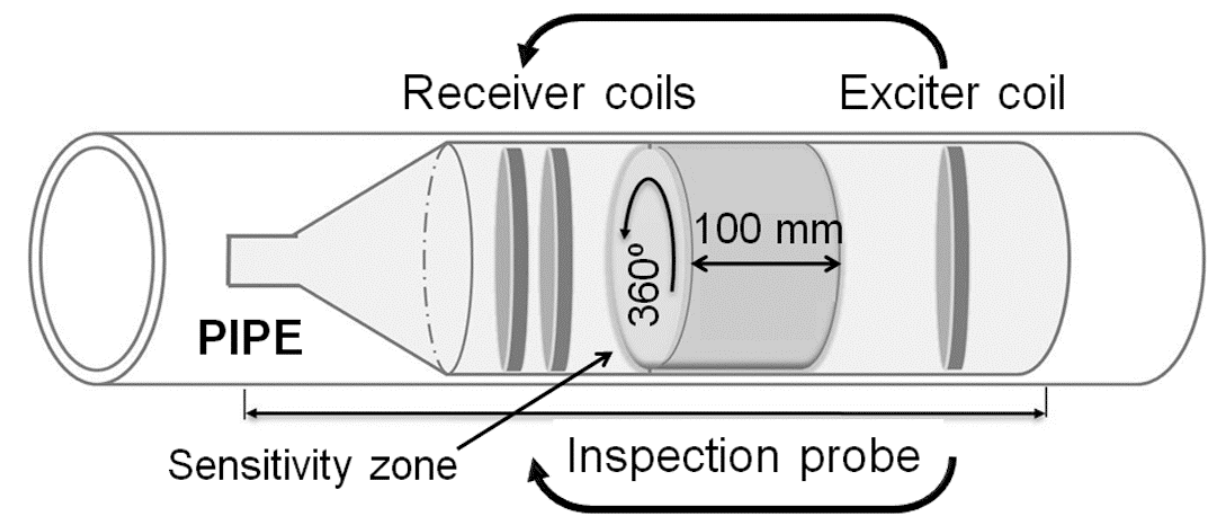

1. Correction of artifacts

2. Estimation of the mean percentage of material loss for $100 \mathrm{~mm}$ by $360^{\circ}$ zones

3. Estimation of the worst percentage of material loss on specific proportions of these

$15100 \mathrm{~mm}$ by $360^{\circ}$ sensitivity zones 


\section{Correction of artifacts}
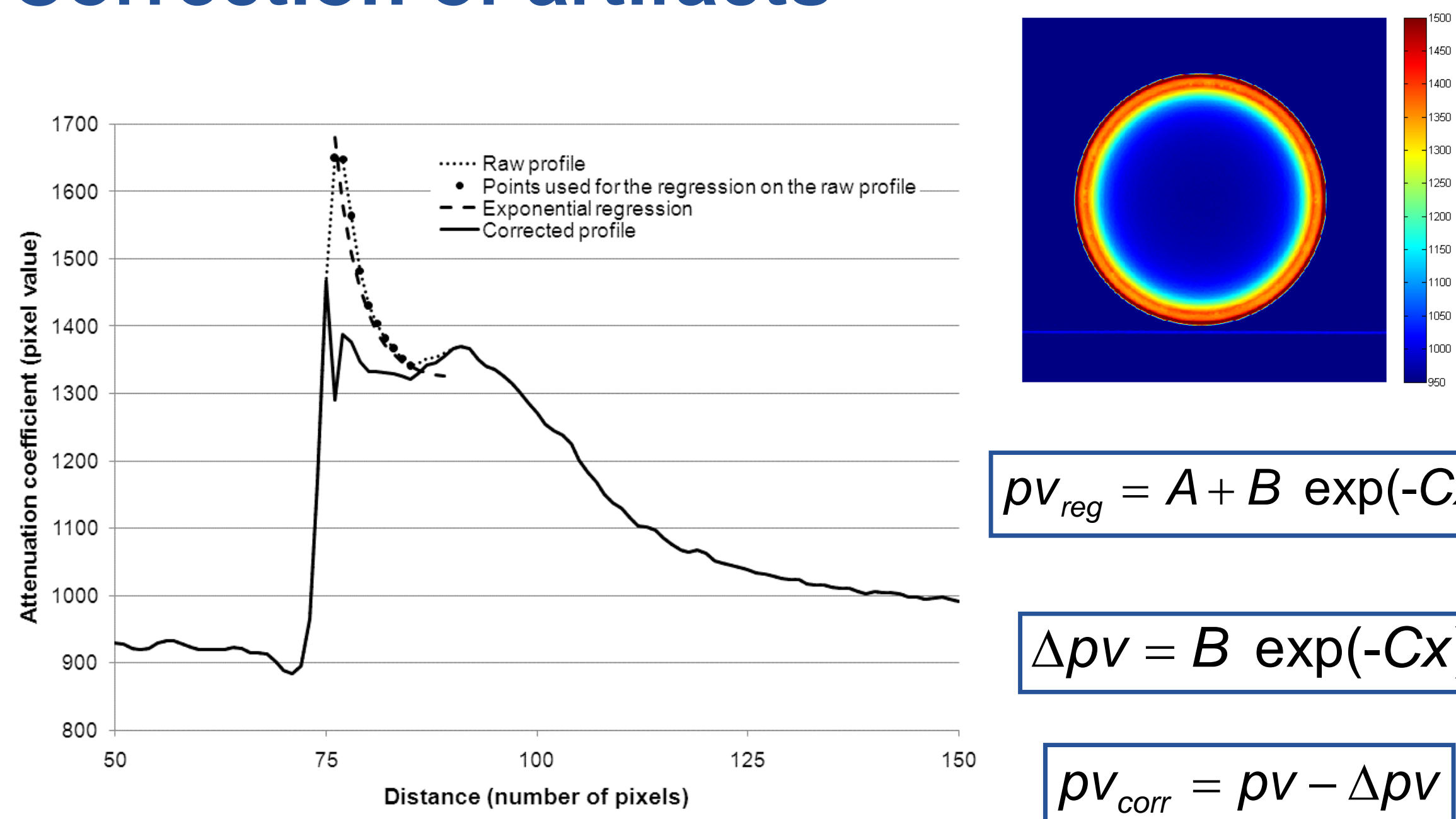

$$
p v_{\text {reg }}=A+B \exp (-C x)
$$$$
\Delta p v=B \exp (-C x)
$$

$$
p v_{\text {corr }}=p v-\Delta p v
$$




\section{Estimation of mean percentage of material loss}

i. Compute mean corrected pixel value across the pipe wall for 180 different angles ( $2^{\circ}$ apart)

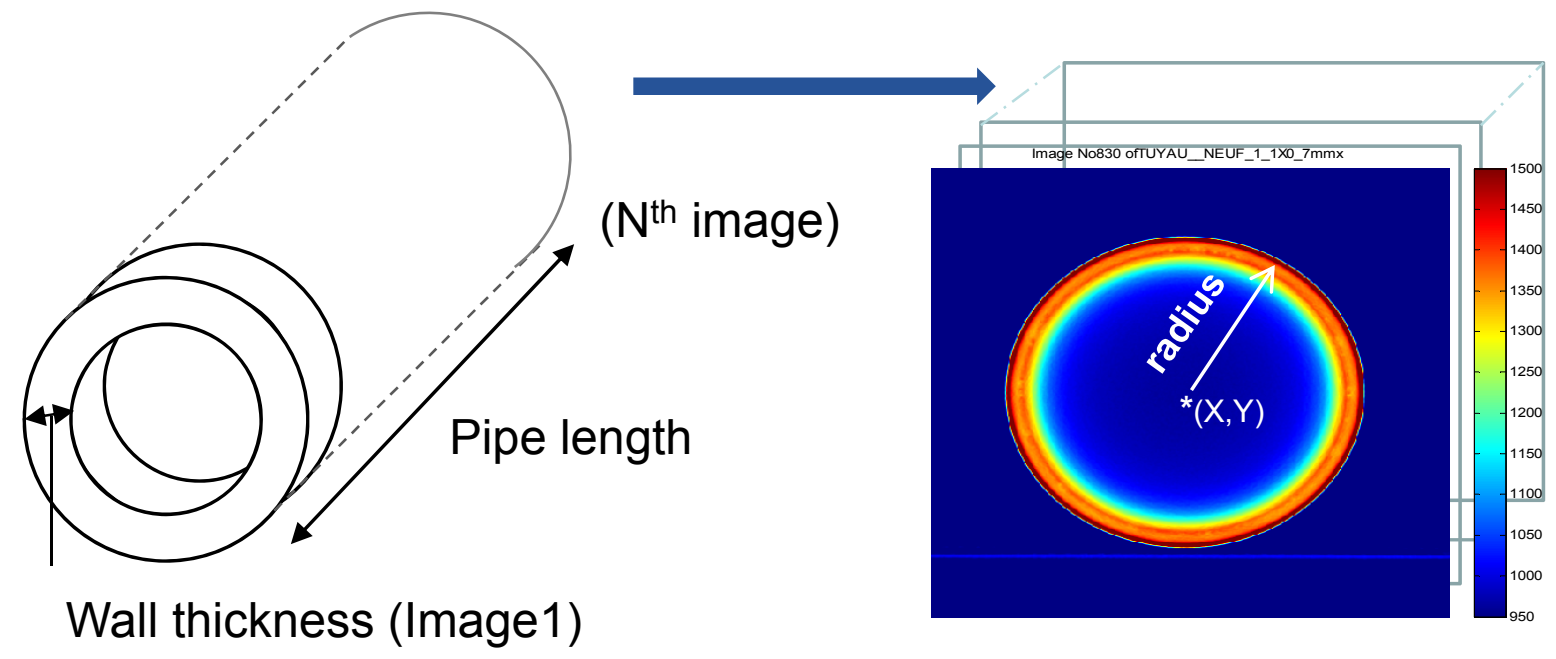

Example for NEW-PIPE

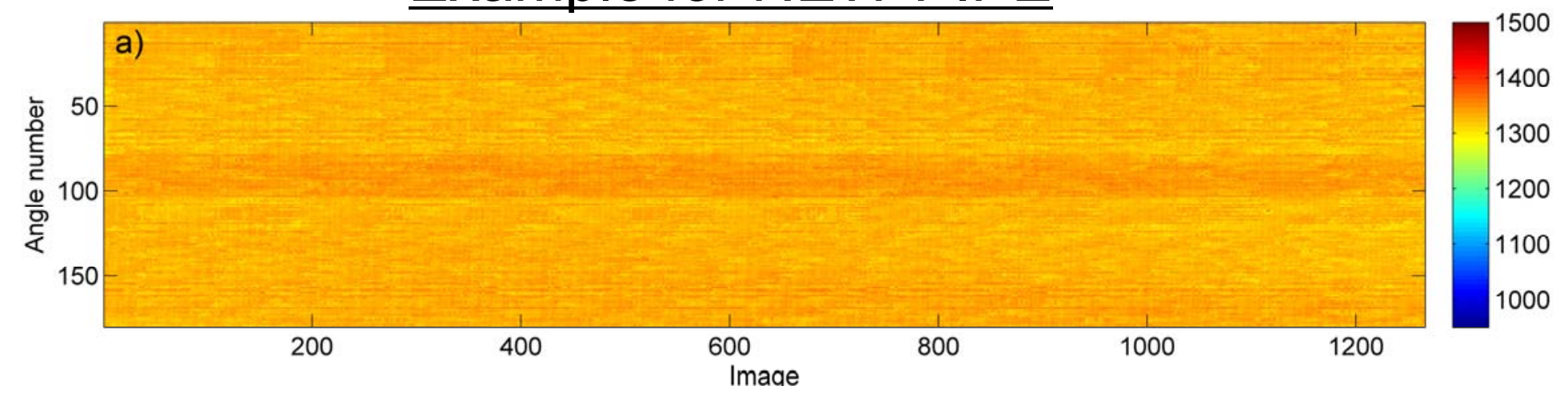




\section{Estimation of mean percentage of material loss}

i. Compute mean corrected pixel value across the pipe wall for 180 different angles ( $2^{\circ}$ apart)

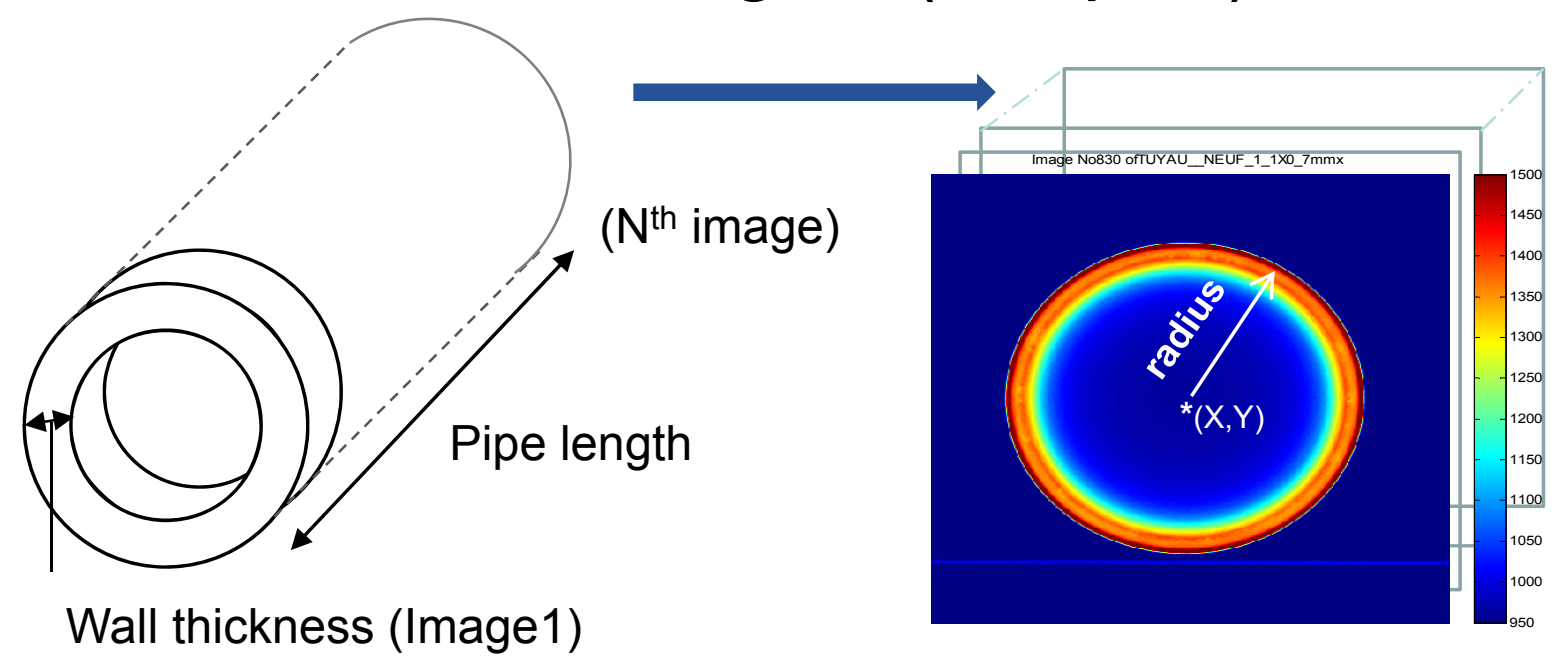

Example for NEW-PIPE

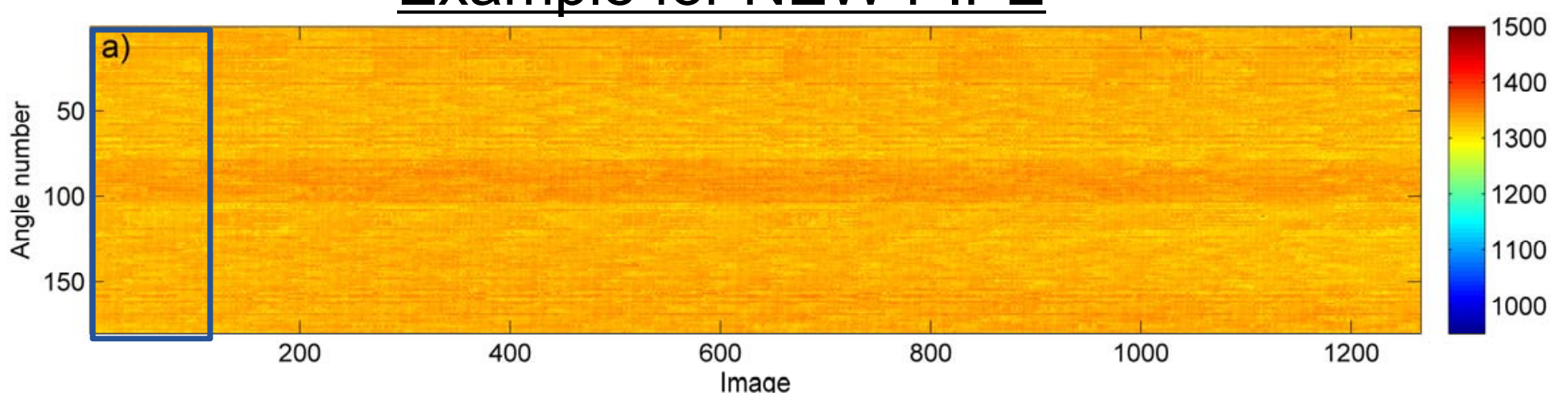

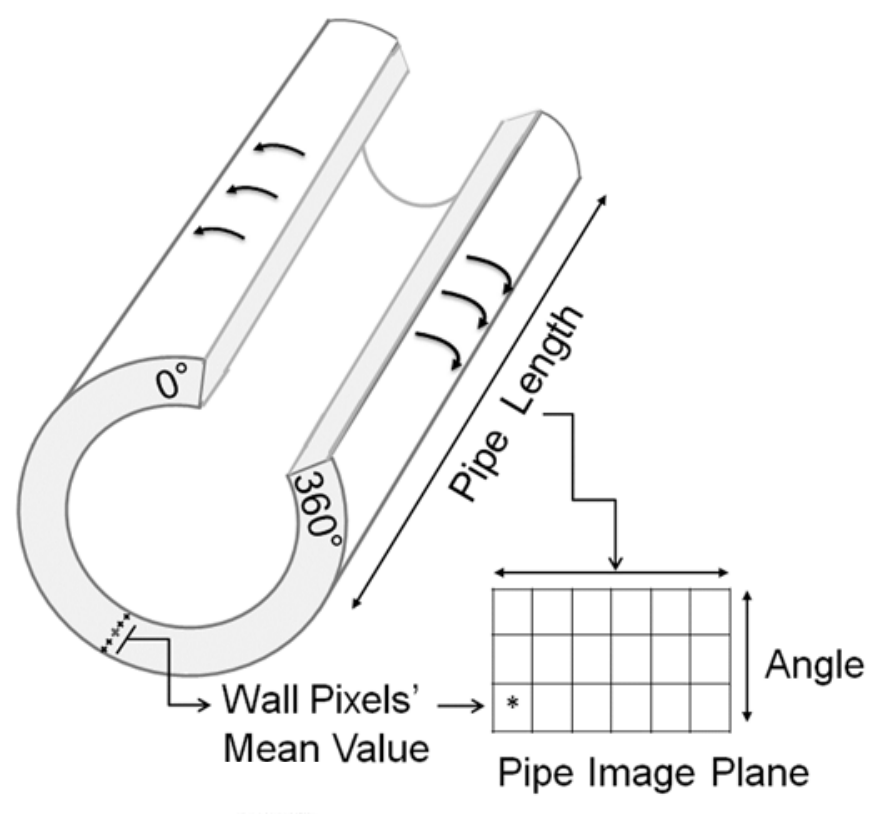

400

00

000 


\section{Estimation of mean percentage of material loss}

ii. Compute percentage of pipe-wall loss for each pixel

$$
\% \text { loss }=\left[1-\left(\frac{p v-p v_{\min }}{p v_{\max }-p v_{\min }}\right)\right] \times 100
$$

iii. Average the percentage of material loss (for $100-\mathrm{mm}$ strips) over all $180-2^{\circ}$ angles $\left(360^{\circ}\right)$
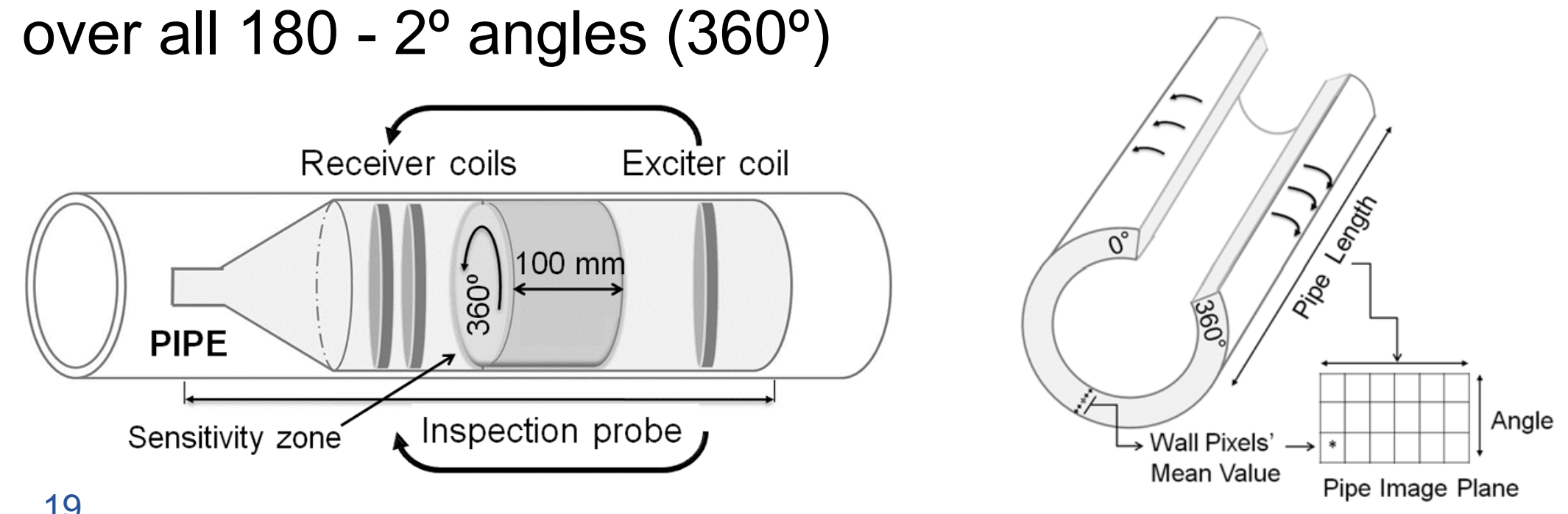


\section{Estimation of mean percentage of material loss}

\section{Example for NEW-PIPE}
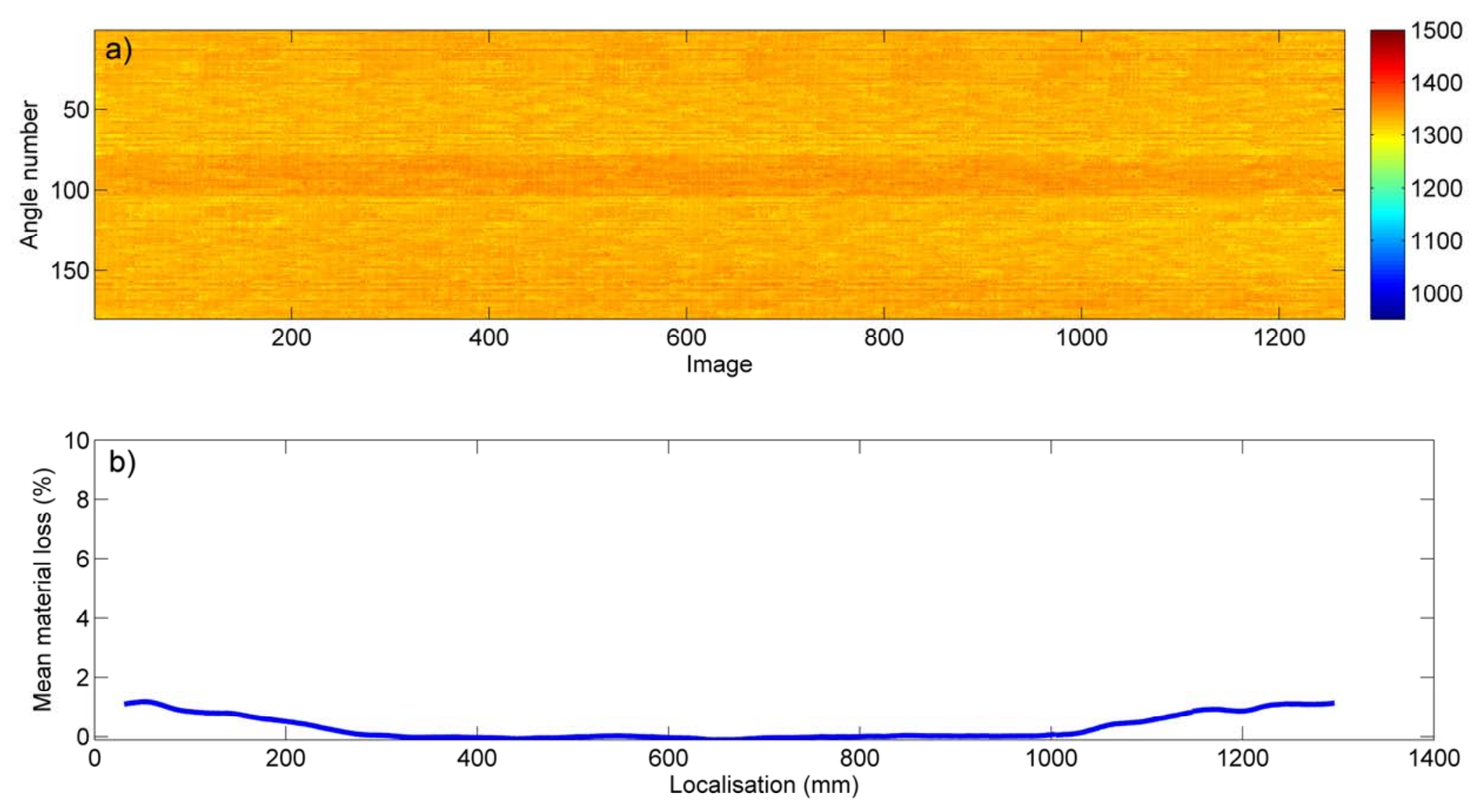


\section{Estimation of mean percentage of material loss}

\section{Example for NEW-PIPE}
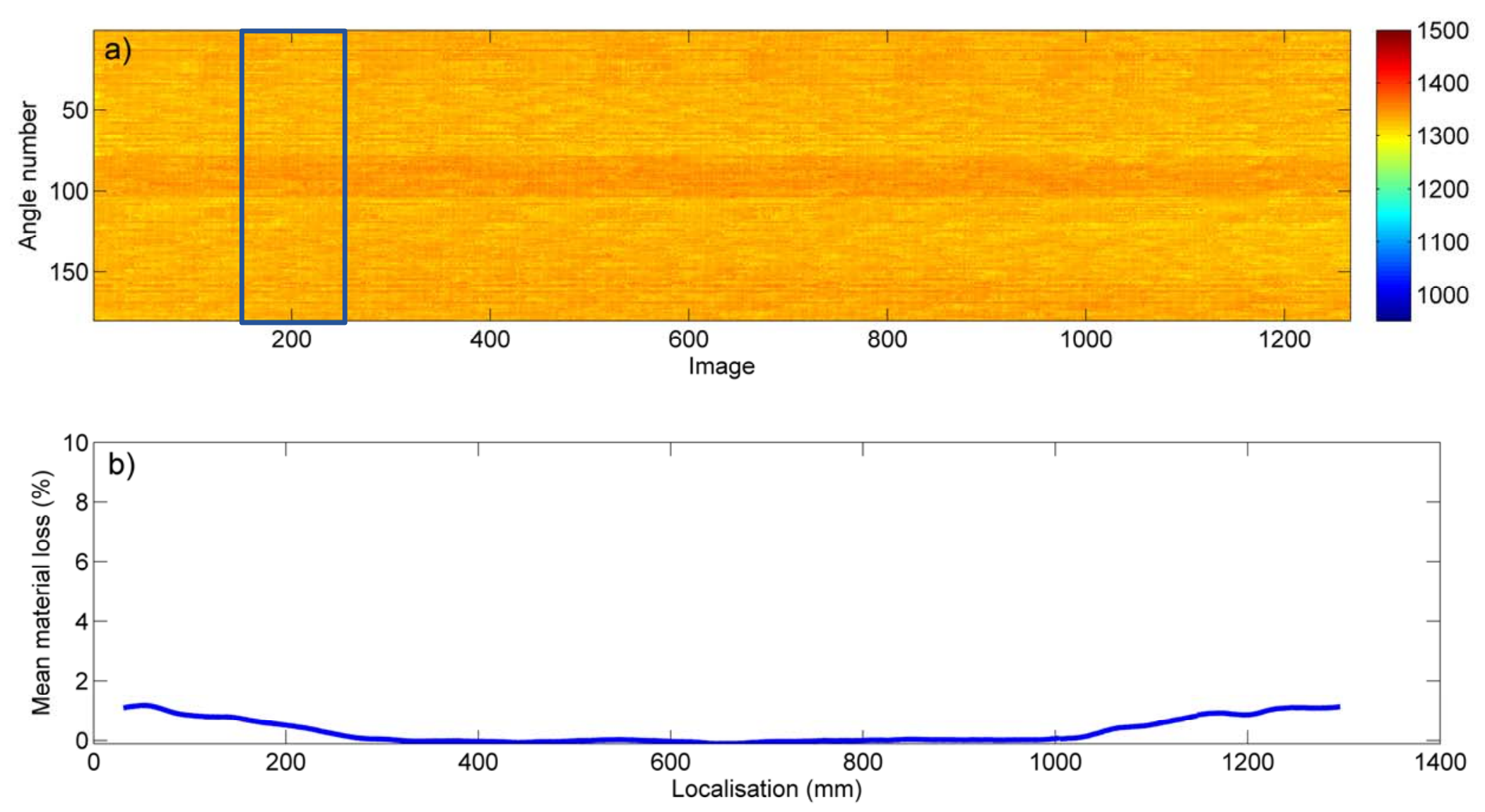


\section{Estimation of worst thickness loss}

\section{Example for SILL-MAG-1A}
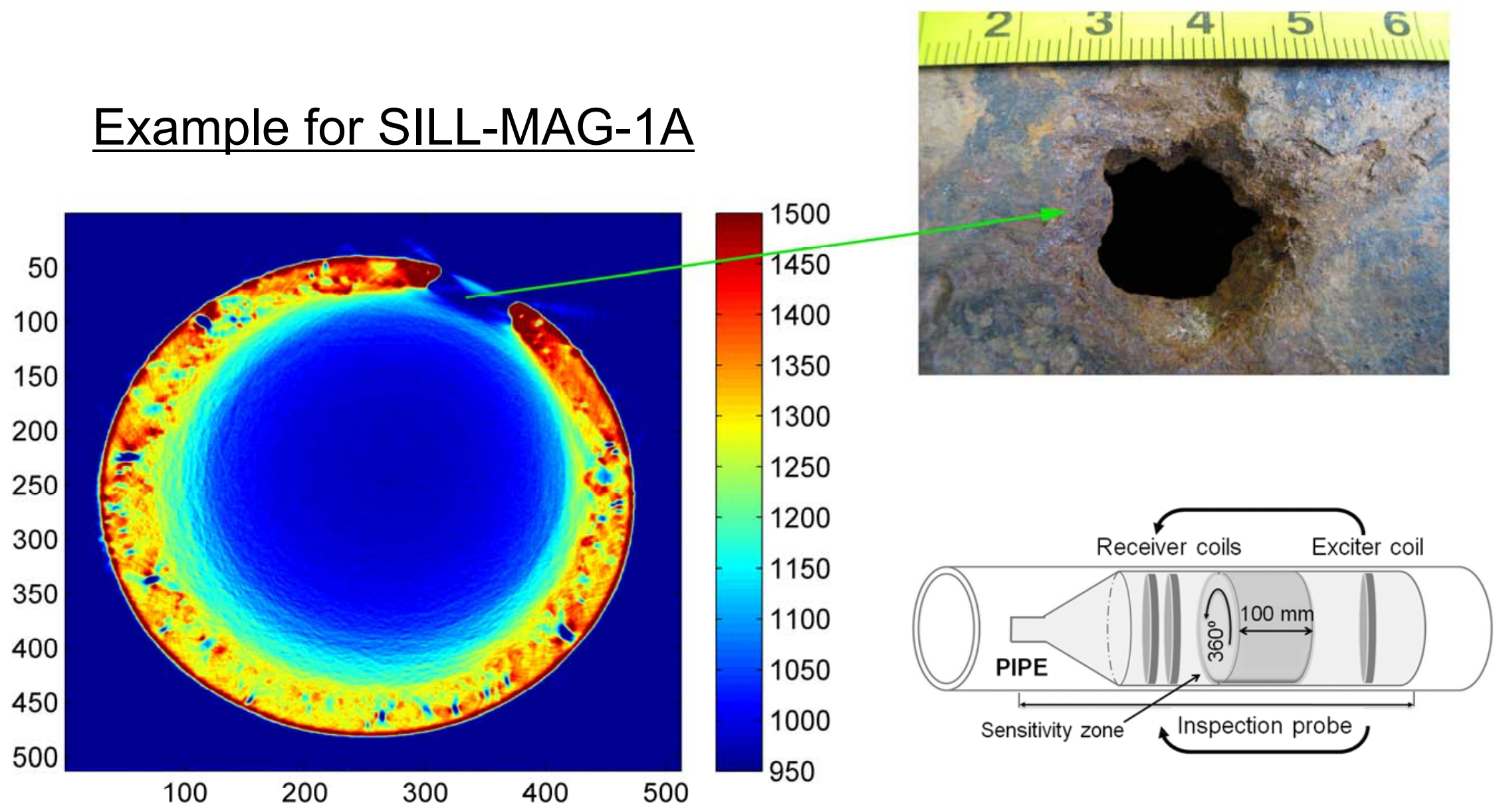


\section{Estimation of worst thickness loss}

\section{Example for SILL-MAG-1A}
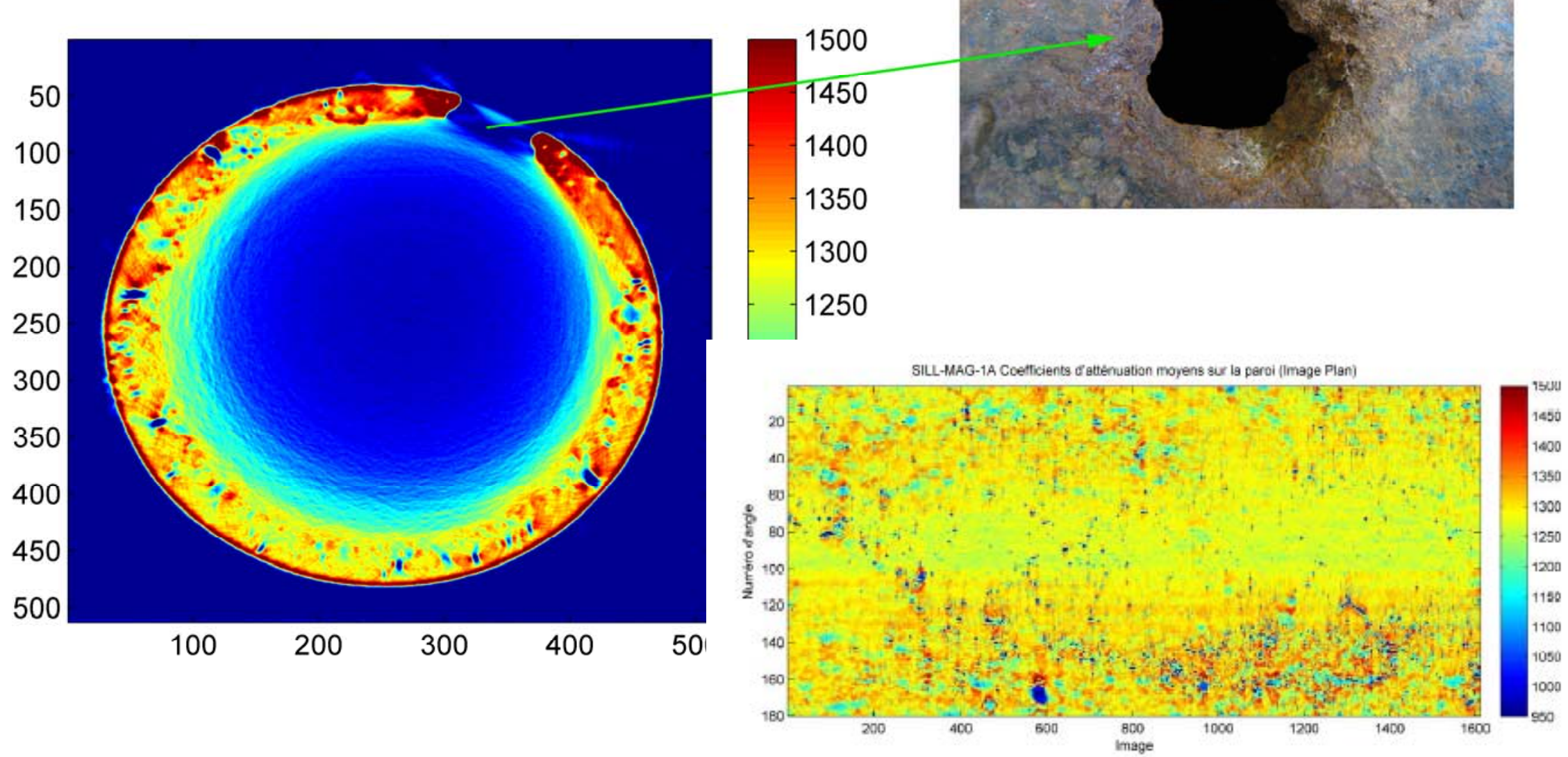


\section{Estimation of worst thickness loss}

Example for SILL-MAG-1A
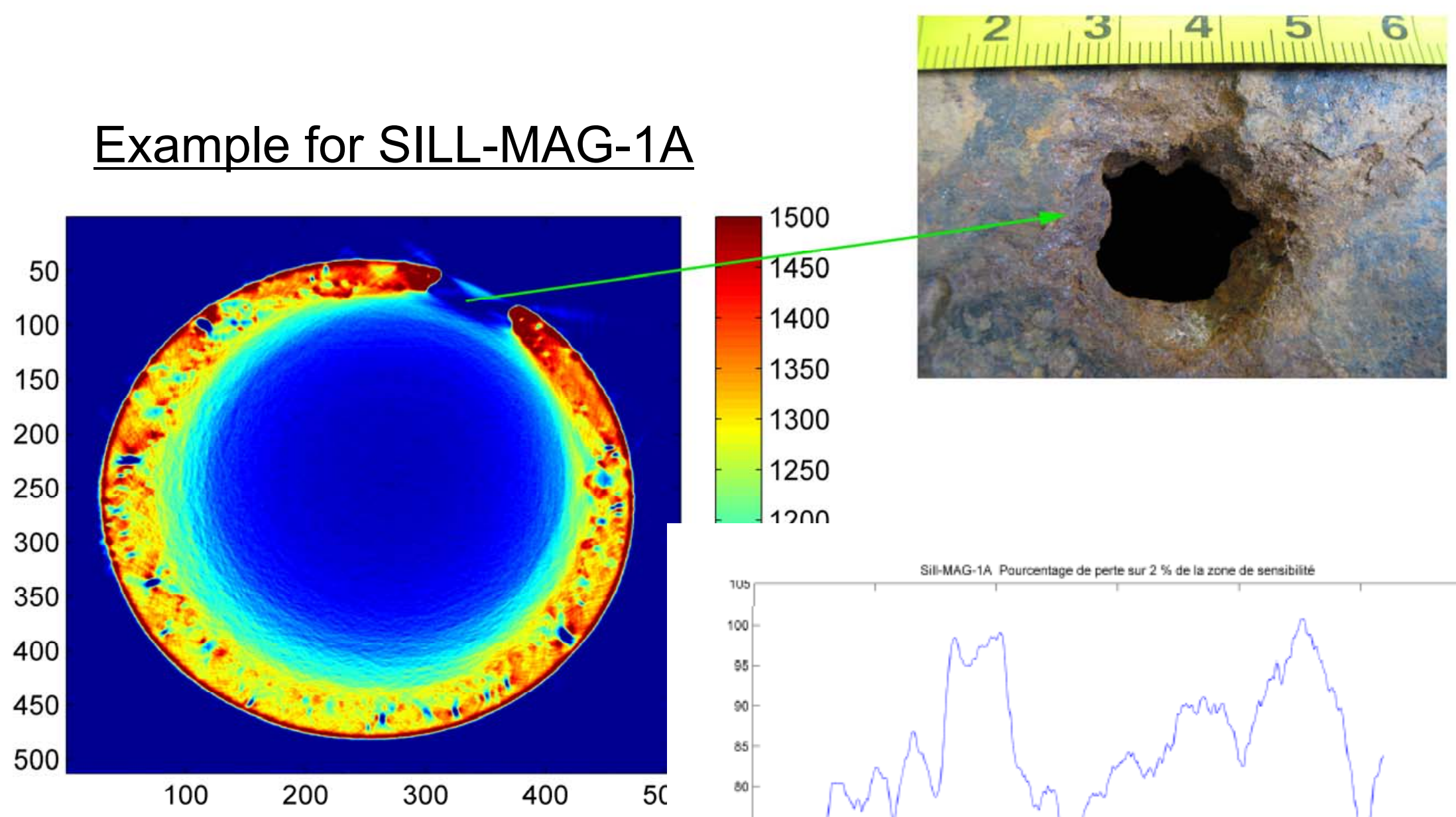

1350

1300

1250

$120 n$

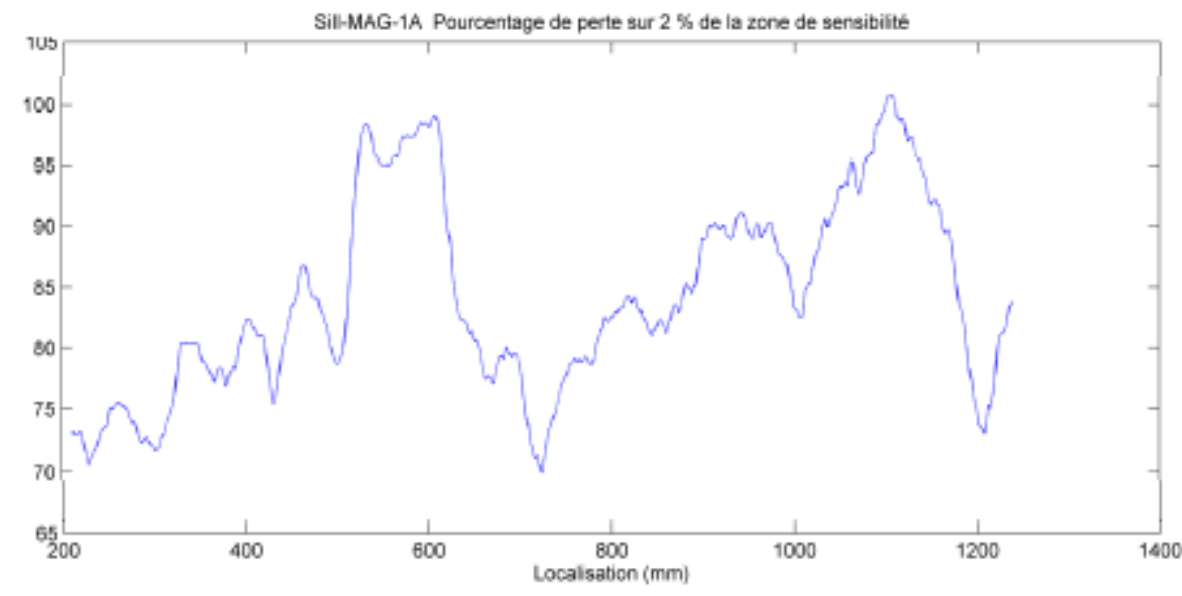

NDT in Canada 2017 Conference (June 6-8, 2017) 


\section{Summary of results}

\begin{tabular}{|c|c|c|c|c|c|c|c|}
\hline \multirow[b]{2}{*}{ Pipe } & \multirow[b]{2}{*}{ Defect } & \multicolumn{3}{|c|}{ CT scan image analysis } & \multicolumn{3}{|c|}{ RFEC Tool } \\
\hline & & $\begin{array}{l}\text { Location } \\
\text { (m) }\end{array}$ & $\begin{array}{c}\text { Thickness } \\
\text { loss (\%) }\end{array}$ & $\begin{array}{c}\text { Sensitivity } \\
\text { zone } \\
\text { coverage } \\
(\%)\end{array}$ & $\begin{array}{l}\text { Location } \\
\text { (m) }\end{array}$ & $\begin{array}{c}\text { Thickness } \\
\text { loss (\%) }\end{array}$ & $\begin{array}{c}\text { Sensitivity } \\
\text { zone } \\
\text { coverage } \\
(\%)\end{array}$ \\
\hline B-MAN-1A & $\begin{array}{l}\# 1 \\
\# 2\end{array}$ & $\begin{array}{l}0.7 \\
1.3\end{array}$ & $\begin{array}{l}18 \\
22\end{array}$ & $\begin{array}{l}13 \\
22\end{array}$ & $\begin{array}{l}\text { n.i. } \\
1.2\end{array}$ & $\begin{array}{l}\text { n.i. } \\
22\end{array}$ & $\begin{array}{c}\text { n.i. } \\
22\end{array}$ \\
\hline B-MAN-1B & $\begin{array}{l}\# 1 \\
\# 2\end{array}$ & $\begin{array}{c}0.7-0.9 \\
1.6\end{array}$ & $\begin{array}{l}16 \\
15\end{array}$ & $\begin{array}{l}13 \\
17\end{array}$ & $\begin{array}{l}0.8 \\
1.6\end{array}$ & $\begin{array}{l}17 \\
28\end{array}$ & $\begin{array}{l}13 \\
17\end{array}$ \\
\hline B-MAN-2A & $\begin{array}{l}\# 1 \\
\# 2\end{array}$ & $\begin{array}{c}0.8 \\
1.2-1.5\end{array}$ & $\begin{array}{l}34 \\
14 \\
\end{array}$ & $\begin{array}{l}13 \\
63\end{array}$ & $\begin{array}{l}\text { n.i. } \\
1.8\end{array}$ & $\begin{array}{l}\text { n.i. } \\
15\end{array}$ & $\begin{array}{l}\text { n.i. } \\
63\end{array}$ \\
\hline B-MAN-2B & $\begin{array}{l}\# 1 \\
\# 2 \\
\# 3\end{array}$ & $\begin{array}{c}0.4 \\
1 \\
1.4\end{array}$ & $\begin{array}{l}20 \\
25 \\
48\end{array}$ & $\begin{array}{l}13 \\
13 \\
13\end{array}$ & $\begin{array}{l}0.4 \\
\text { n.i. } \\
1.3\end{array}$ & $\begin{array}{l}23 \\
\text { n.i. } \\
26\end{array}$ & $\begin{array}{c}<13 \\
\text { n.i. } \\
13\end{array}$ \\
\hline LHSTCH-MC & $\begin{array}{l}\# 1 \\
\# 2 \\
\end{array}$ & $\begin{array}{l}0.1 \\
0.7\end{array}$ & $\begin{array}{l}27 \\
20 \\
\end{array}$ & $\begin{array}{l}13 \\
24 \\
\end{array}$ & $\begin{array}{l}\text { n.i. } \\
0.7\end{array}$ & $\begin{array}{l}\text { n.i. } \\
38\end{array}$ & $\begin{array}{l}\text { n.i. } \\
24\end{array}$ \\
\hline $\begin{array}{l}\text { LHSTCH- } \\
\text { HOP }\end{array}$ & $\begin{array}{l}\# 1 \\
\# 2\end{array}$ & $\begin{array}{l}0.2 \\
1.4\end{array}$ & $\begin{array}{l}17 \\
12\end{array}$ & $\begin{array}{l}13 \\
13\end{array}$ & $\begin{array}{l}\text { n.i. } \\
1.4\end{array}$ & $\begin{array}{l}\text { n.i. } \\
20\end{array}$ & $\begin{array}{l}\text { n.i. } \\
<13\end{array}$ \\
\hline
\end{tabular}

n.i.: not identified 


\section{Conclusions}

- Similar results for both techniques:

- but RFEC tool: thickness loss $\geq 15 \%$ when averaged on the $13 \%$ most corroded area of the tool's sensitivity zone

- RFEC tool provides reliable information on the main corrosion defects and thus on the general structural state of water pipes

- RFEC tool cannot identify small corrosion pits:

- could cause leaks and even initiate larger corrosion areas

- better detected by leak detection methods (e.g. acoustic)

- Further tests required (more pipes, lined or coated pipes, ductile iron pipes) 


\section{Questions ?}
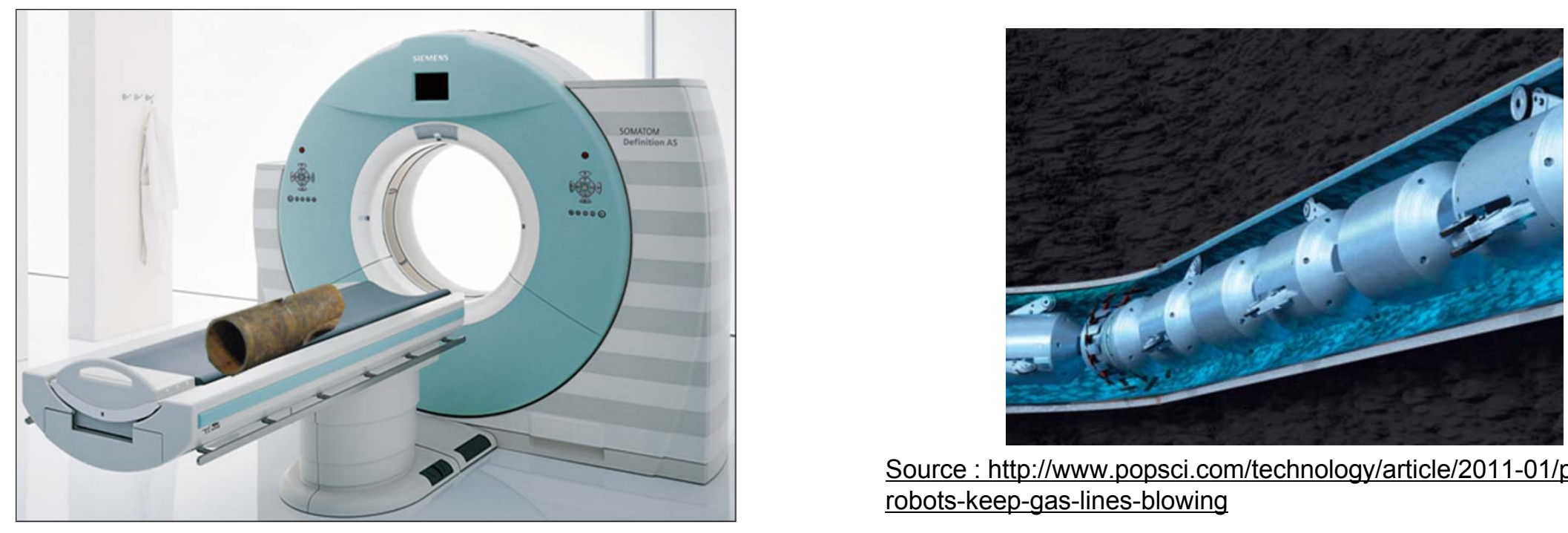

Source : http://www.popsci.com/technology/article/2011-01/pigrobots-keep-gas-lines-blowing 


\begin{tabular}{|l|c|c|c|}
\hline \multirow{2}{*}{ Name } & $\begin{array}{c}\text { Pixel size } \\
(\mathrm{mm})\end{array}$ & $\begin{array}{c}\text { Slice } \\
\text { thickness }\end{array}$ & $\begin{array}{c}\text { Spacing between } \\
\text { slices }\end{array}$ \\
\hline NEW-PIPE & 0.492 & $(\mathrm{~mm})$ & 0.7 \\
\hline SILL-MAG-1A & 0.517 & 1 & 0.7 \\
\hline B-MAN-1A & 0.492 & 1 & 0.7 \\
\hline B-MAN-1B & 0.492 & 1 & 0.7 \\
\hline B-MAN-2A & 0.492 & 1 & 0.7 \\
\hline B-MAN-2B & 0.492 & 1 & 0.7 \\
\hline LHSTCH-MC & 0.449 & 1 & 0.7 \\
\hline LHSTCH-HOP & 0.431 & 1 & 1.0 \\
\hline
\end{tabular}



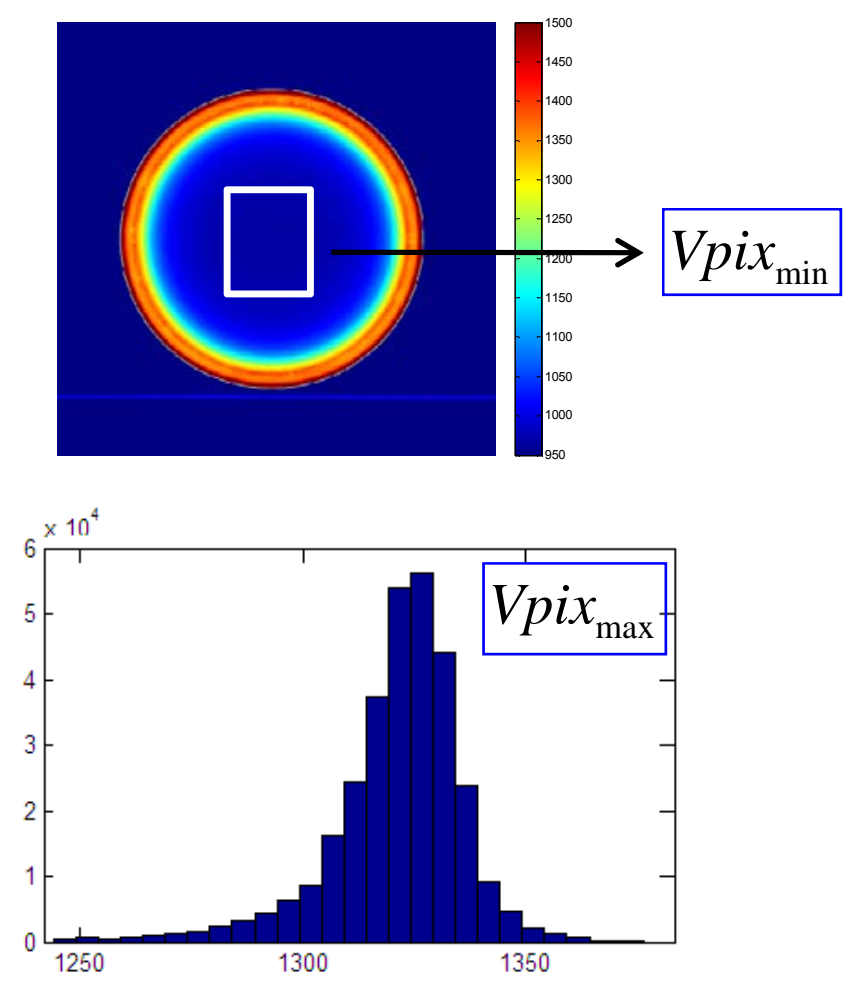